(1)

CrossMark

\title{
MicroRNAs in COPD: small molecules with big potential
}

\author{
Brian D. Hobbs (10) and Kelan G. Tantisira
}

Affiliation: Channing Division of Network Medicine and Division of Pulmonary and Critical Care Medicine, Brigham and Women's Hospital, Boston, MA, USA.

Correspondence: Brian D. Hobbs, Brigham and Women's Hospital, Channing Division of Network Medicine and Division of Pulmonary and Critical Care Medicine, 181 Longwood Ave, Boston, MA 02115, USA. E-mail: rebdhachanning.harvard.edu

@ERSpublications

MicroRNA research in COPD can help explain the observed heterogeneity, not only in clinical presentation, but also in treatment response, particularly as it relates to inhaled corticosteroids http://ow.ly/HbYo30o7lit

Cite this article as: Hobbs BD, Tantisira KG. MicroRNAs in COPD: small molecules with big potential. Eur Respir J 2019; 53: 1900515 [https://doi.org/10.1183/13993003.00515-2019].

Micro ribonucleic acids (microRNAs or miRNAs) are small ( 22 nucleotide in length) non-protein-coding regulatory RNAs that have the potential to impact the development and progression of nearly all human diseases through interactions with messenger RNAs (mRNAs) [1]. MicroRNAs are transcribed from non-protein-coding regions of the genome into hairpin structures in the nucleus of cells and then are exported into the cytoplasm. The mature miRNA complexes can act within the cell or be secreted into the extracellular compartment to facilitate cell-cell communication. With partial miRNA-mRNA pairing, the target mRNA will be translationally repressed (less protein is made from the mRNA). With more complete miRNA-mRNA pairing, the mRNA will be sliced and silenced (i.e. no protein will be made) (figure 1). Any single miRNA can potentially target and repress hundreds of mRNAs [2]. These broad networks of miRNA-mRNA interactions allow a single miRNA to potentially impact a broad range of biological processes. Knockdown of miRNAs in mice has illustrated the potential impact of miRNAs in both contributing to and protecting from respiratory diseases [1].

Chronic obstructive pulmonary disease (COPD) is a heterogenous disease, not only in clinical presentation [3], but also in the variable response to therapy, particularly inhaled corticosteroids (ICS) [4]. Over the past $\sim 10$ years, microRNA research in COPD has advanced our understanding of the role miRNAs may play in contributing to the phenotypic heterogeneity of COPD. MicroRNA expression studies in lung tissue, specific lung cell types, peripheral blood and immune cells have associated a number of miRNAs with COPD [5-7] as well as with COPD-related phenotypes, such as emphysema [8] and the asthma-COPD overlap syndrome [9]. Beyond evaluating miRNAs in isolation, several investigators have leveraged the negative regulatory relationship between miRNAs and mRNAs to build networks of miRNA-mRNA interactions that are associated with COPD [10]. Network-based investigations have the advantage of offering more immediate follow-up of the functional implications of miRNAs acting through specific mRNAs in contributing to COPD pathobiology. Additional studies of microRNA differential expression and miRNA-mRNA network interactions in COPD and COPD-related phenotypes are needed to validate prior findings and help describe the functional contribution of miRNAs to COPD phenotypic heterogeneity. Despite growing research in contributions of microRNAs to COPD pathobiology, little research to date has addressed the variable responses of COPD patients to available therapeutics. 

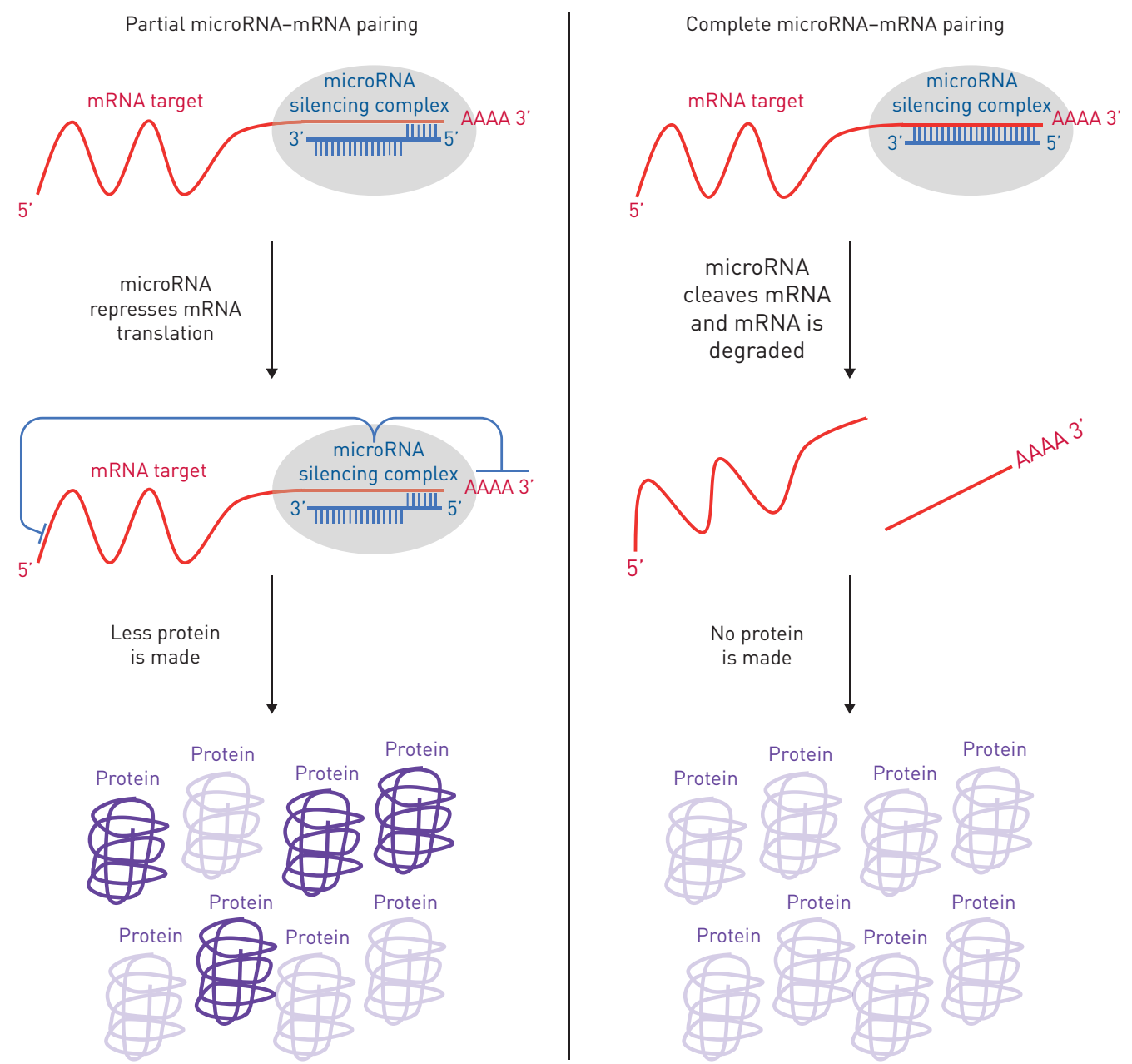

FIGURE 1 MicroRNA-mRNA interactions. With partial microRNA-mRNA pairing, the translation of mRNA to protein is repressed through a variety of mechanisms. With complete complementarity of microRNA and mRNA, the mRNA will be cleaved and degraded. Thus, the target mRNA is silenced and cannot make protein. Reproduced with permission from the publisher [1].

In the current issue of the European Respiratory Journal, FAIZ et al. [11] report the results of a genome-wide microRNA analysis assessing the changes in microRNA profiles of bronchial biopsies from persons with moderate-to-severe COPD following 6- and 30-month treatment with an ICS. The investigators performed staged investigations of the association of 230 measured miRNAs with ICS treatment, showing four miRNAs altered in the bronchial biopsies of persons with COPD following 6- and 30-month treatment with ICS. Of these four miRNAs, miR-320d was highlighted, as multiple lines of corollary external evidence reinforced the potential role of miR-320d in modulating the therapeutic response to ICS. First, miR-320d showed increased expression in bronchial biopsies after 6- and 30-month ICS treatment compared to placebo. Further, the predicted mRNA targets of miR-320d were significantly decreased at 6 months following ICS treatment. In a separate dataset, the predicted mRNA targets of miR-320d were increased in bronchial brushings of COPD cases compared to controls, which suggests a possible therapeutic role for suppression of miR-320d mRNA targets. In vitro fluticasone treatment of epithelial cells demonstrated a significant miR-320d upregulation, which was consistent with the in vivo microarray results. A pathway analysis suggested miR-320d mRNA targets were enriched in gene sets associated with cytokine production. Further, a network-based analysis of publicly available data highlighted IL-1 $\beta$ as a "hub" in a network of genes associated with cigarette smoke exposure. These bioinformatic analyses directed a series of in vitro functional studies and allowed the investigators to propose that in the setting of cigarette smoke exposure, miR-320d has an anti-inflammatory role mediated by suppression of NF- $\kappa \mathrm{B}$ activity.

The work presented by FAIZ et al. [11] has several strengths. First, the investigators utilised a series of staged investigations (including validation in both humans and cell culture) to avoid carrying false-positive associations from the primary genome-wide microRNA analysis into downstream functional analyses. 
Second, by evaluating overlapping microRNA and mRNA data, the authors were able to capture regulatory consequences of differences in miRNA expression, which help them to design functional studies for miR-320d. Finally, the investigators carried their primary findings from initial discovery, through functional studies, to a proposed mechanism of action. One limitation of the proposed mechanism of action of miR-320d is the dependence on cigarette smoke-induced inflammation, in which not all subjects in the discovery of miR-320d association with ICS treatment were current smokers. A stratified analysis of current and former smokers would help to determine if the observed differences in miR-320d expression between placebo and ICS treatment is modified by smoking. From a broader perspective, none of the microRNAs differentially expressed by ICS in the FAIZ et al. [11] study overlap with those from bronchial cells of ICS-treated asthmatic patients $[12,13]$, though differences in study design, sample size, and ICS type, dose and duration were present. The question remains, is there a global set of miRNAs involved in the regulation of glucocorticoid sensitivity and resistance, or will disease-specific steroid "insensitivity" states necessitate distinct miRNA-mediated therapeutics?

As noted by the investigators, further functional work, possibly in mouse models of COPD, needs to be performed to investigate the immunomodulatory role of miR-320d. Short of further validation and functional work, the proposed function of miR-320d in ICS-mediated immunomodulation by FAIz et al. [11] lays a foundation for translational research. For instance, a panel of miR-320d gene targets is a potential biomarker for ICS response, in which the relative upregulation of miR-320d targets in COPD patients might predict a more robust response to ICS. Alternatively, miR-320d could be directly assessed, in which relatively higher levels of miR-320d in COPD patients may predict a poor response to ICS. In the future, if the role of miR-320d related response to ICS is confirmed in the airways and characterised, a miR-320d mimic could be investigated as either an alternative or adjunctive therapy to ICS in COPD.

Conflict of interest: None declared.

Support statement: B.D. Hobbs is supported by NIH K08 HL136928 and the Parker B Francis Research Opportunity Award. K.G. Tantisira is supported by NIH R01 HL127332 and R01 HL129935. Funding information for this article has been deposited with the Crossref Funder Registry.

\section{References}

Bartel DP. Metazoan MicroRNAs. Cell 2018; 173: 20-51.

2 Hendrickson DG, Hogan DJ, McCullough HL, et al. Concordant regulation of translation and mRNA abundance for hundreds of targets of a human microRNA. PLoS Biol 2009; 7: e1000238.

3 Vogelmeier CF, Criner GJ, Martinez FJ, et al. Global Strategy for the Diagnosis, Management, and Prevention of Chronic Obstructive Lung Disease 2017 Report. GOLD Executive Summary. Am J Respir Crit Care Med 2017; 195: 557-582.

4 Ernst P, Saad N, Suissa S. Inhaled corticosteroids in COPD: the clinical evidence. Eur Respir J 2015; 45: 525-537.

5 Molina-Pinelo S, Pastor MD, Suarez R, et al. MicroRNA clusters: dysregulation in lung adenocarcinoma and COPD. Eur Respir J 2014; 43: 1740-1749.

6 Kim WJ, Lim JH, Hong Y, et al. Altered miRNA expression in lung tissues of patients with chronic obstructive pulmonary disease. Mol Cell Toxicol 2017; 13: 207-212.

7 Wang R, Xu J, Liu H, et al. Peripheral leukocyte microRNAs as novel biomarkers for COPD. Int J Chron Obstruct Pulmon Dis 2017; 12: 1101-1112.

8 Christenson SA, Brandsma CA, Campbell JD, et al. miR-638 regulates gene expression networks associated with emphysematous lung destruction. Genome Med 2013; 5: 114.

9 Lacedonia D, Palladino GP, Foschino-Barbaro MP, et al. Expression profiling of miRNA-145 and miRNA-338 in serum and sputum of patients with COPD, asthma, and asthma-COPD overlap syndrome phenotype. Int J Chron Obstruct Pulmon Dis 2017; 12: 1811-1817.

10 Qian Y, Mao ZD, Shi YJ, et al. Comprehensive analysis of miRNA-mRNA-lncRNA networks in non-smoking and smoking patients with chronic obstructive pulmonary disease. Cell Physiol Biochem 2018; 50: 1140-1153.

11 Faiz A, Steiling K, Roffel MP, et al. Effect of long-term corticosteroid treatment on microRNA and gene-expression profiles in COPD. Eur Respir J 2019; 53: 1801202.

12 Williams AE, Larner-Svensson H, Perry MM, et al. MicroRNA expression profiling in mild asthmatic human airways and effect of corticosteroid therapy. PLoS One 2009; 4: e5889.

13 Solberg OD, Ostrin EJ, Love MI, et al. Airway epithelial miRNA expression is altered in asthma. Am J Respir Crit Care Med 2012; 186: 965-974 